\title{
Influence of Soft Reduction on the Fluid Flow, Porosity and Center Segregation in CC High Carbon- and Stainless Steel Blooms
}

\author{
Bo ROGBERG ${ }^{1 * *}$ and Lina $\mathrm{EK}^{21}$ \\ 1) KTH Royal Institute of Technology, SE-100 44, Stockholm, Sweden. \\ 2) Sandvik Materials Technology, SE-811 81 Sandviken, Sweden.
}

(Received on September 9, 2017; accepted on November 9, 2017)

\begin{abstract}
Mechanical soft reduction, MSR, is today an established technique for reducing center segregation and center porosity in the continuous casting of blooms. The bloom cross section is reduced by pinch rollers to compensate for the downward liquid flow in the mushy zone due to solidification shrinkage. In the literature, results are reported on how much MSR quantitatively affects the center segregation, but not on how it influences the liquid flow velocities.

An analytical mathematical model has been developed to calculate the liquid flow velocity along the strand taking into account the effect of height reduction at each pinch roller and the solidification shrinkage. The model considers how much of the total area reduction contributes to an area reduction of the liquid in the center and thus how it affects the flow velocities. The model is easy to apply and brings deeper understanding of the effect of MSR. Calculated results from different scenarios show that the area reduction should increase closer to the crater end. MSR trials on a high carbon steel grade show that the center segregation decreases in proportion to the amount of reduction and clarify the importance of applying a pinch roller just before the crater end. Elongations of the strand above $\sim 1 \%$ gave inner cracks in this case. MSR of only $2 \%$ height reduction on a stainless steel bloom, type AISI 316, gave a substantial closure of visual center porosity on inspected longitudinal samples.
\end{abstract}

KEY WORDS: continuous casting; soft reduction; center segregation; fluid flow.

\section{Introduction}

In the continuous casting of blooms, severe macrosegregation and porosity arises along the center axis. To improve the center quality, modern blooms machines ${ }^{1-3)}$ are equipped with a reduction zone for Mechanical Soft $\underline{\text { Reduction, MSR, }}$ consisting of 4-10 pinch rollers. A small height reduction of the strand at each pinch roller is carried out to reduce the liquid fraction in mushy zone in order to compensate for the solidification shrinkage. This reduces the downward liquid flow velocity relative to the solid phase in the casting direction.

The process of choosing optimal process parameters for MSR is still very much one of trial and error, as pointed out by Wu et al. ${ }^{4)}$ Height reduction in [mm] for each pinch roller is a frequently used parameter for blooms and is usually in the range of $1-12 \mathrm{~mm}$. However, the parameter is not related to the absolute height of the bloom, which is a limitation. Ogibayashi et al. ${ }^{5)}$ defined the amount of reduction gradient as the total height reduction in [mm] divided by the length of the reduction zone in [m]. Multiplying the reduction gradient with the casting speed, they obtained the reduction rate in $[\mathrm{mm} / \mathrm{min}]$. Those process parameters were then correlated to the center segregation. However,

\footnotetext{
* Corresponding author: E-mail: brogberg@kth.se

DOI: http://dx.doi.org/10.2355/isijinternational.ISIJINT-2017-534
}

the parameter values are then dependent on the distance between pinch rollers and are therefore not directly applicable to other designs of reduction zones.

Practical tests have shown that a large height reduction value creates internal cracks in the two-phase region in the center. $^{2,6-9)}$ This is a limiting factor and to come around this problem the number of pinch rollers installed on modern machines has increased to enable reduction in small steps.

The numerically calculated fraction of solidified phase, $f_{s}$, along the center axis is another important process parameter. MSR is usually recommended ${ }^{2,5)}$ to be applied in the range of $f_{s}=0.2-0.9$. The challenge is to find the optimum casting speed to adjust the position of the mushy zone in the center to fit to the reduction zone location in order to obtain as low segregation as possible without crack formation. Cracks develop especially at low $f_{s}$ values at the entrance of the reduction zone.

Results in the literature usually focus on how MSR reduces the center segregations but not how it quantitatively affects liquid velocities, which is the primary objective. Already in the early 1980s, Miyazawa and Schwerdtfeger ${ }^{11)}$ calculated the liquid flow velocity in relation to the solid phase in the mushy zone in slabs due to solidification shrinkage. It was shown that the melt velocity component in the casting direction is about 100 times larger than the velocity component in the horizontal direction towards the outer surface. In the extreme case, that the horizontal perme- 
ability is much smaller than the permeability in the casting direction, the liquid flows only in the casting direction.

It is shown ${ }^{12)}$ that, during stationary conditions in a fully developed mushy zone along the strand, the average downward liquid flow velocity relative to the solid, $v_{l}^{\text {rel }}$, for an arbitrary strand cross-section is proportional to the casting speed, $v_{\text {cast }}$, and solidification shrinkage, $\beta$ :

$$
v_{l}^{\text {rel }}=v_{\text {cast }} \cdot \beta
$$

The high casting speed in continuous casting (when compared to ingot casting), results in high $v_{l}^{\text {rel }}$ values according to Eq. (1) and an extended mushy zone in the center. This is the main cause for the formation of center segregations and porosities in blooms.

Along the strand center, the downward flow velocity vector is highest due to the lowest resistance of the mushy zone and that creates the main problem with macrosegregation. Closer to the surface the downward velocity vector decreases gradually. Kajitani et al. ${ }^{13)}$ calculated, by numerical modelling of a slab, the ratio of the center flow velocity vector to the average, $v_{l}^{\text {rel }}$, to be $1.6 / 0.95=1.68$. An evaluation of the corresponding modelling results by Miyazawa and Schwerdtfeger ${ }^{11}$ gives the ratio 1.3 . For blooms and billets, it could be expected that the ratio should be even higher due to the 2D-solidification of a square/rectangular cross section. This is also indicated by sulphur-doped nail experiment on billets ${ }^{14)}$ where a rough estimation gives the ratio $\sim 2$. This higher value could explain why billets and blooms exhibits more severe center segregations than slabs.

In the present study, an analytical model is developed that quantitatively predicts how $v_{l}^{\text {rel }}$ is affected by MSR and solidification shrinkage. Different cases of height reductions and pinch roller positions on $v_{l}^{\text {rel }}$ are calculated in order to increase the fundamental understanding of the effect of MSR and how to design a reduction zone. Results from MSR experiments on a high carbon steel grade and a stainless steel grade, to decrease the center segregation and pore formation, are also presented. The results are analyzed using the analytical model. The development of half-way cracks, due to the height reduction, is pointed out as a severe limiting factor and is dependent on the hot ductility properties of the steel grade.

\section{Analytical Model for the Liquid Flow Velocity}

The analytical mathematical model consists of two parts. In the first part, the relation between area reduction and the amount of liquid area change in the center, affecting the liquid flow velocity, is estimated. Thereafter, in the second part, derivations are given of how $v_{l}^{\text {rel }}$ varies along the strand due to MSR and solidification shrinkage.

\subsection{Relation between the Strand Area Reduction and Liquid Area Change}

The analytical model is based on the schematic drawing in Fig. 1. The strand height, $h$, is reduced between the two pinch rollers, R, from the height before, $h^{b}$, to the height after, $h^{a}$. The solid and liquid phases are shown, as well as the casting direction. The liquid and solid flow velocities, $v_{l}$ and $v_{s}$, before and after the height reduction are also indicated in the figure.

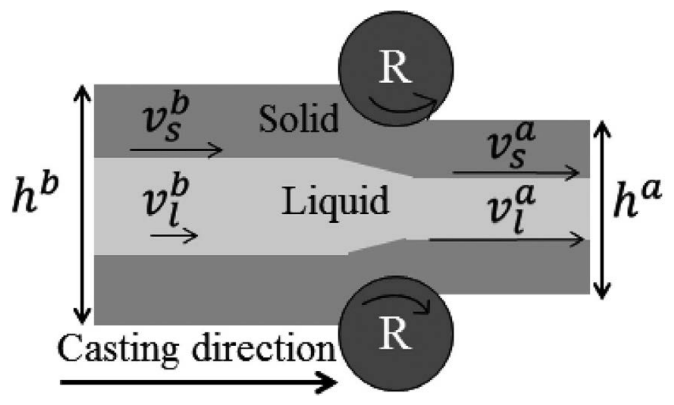

Fig. 1. Schematic view of the strand along the center line with pinch roller and indication of solid and liquid flow velocities before and after deformation.

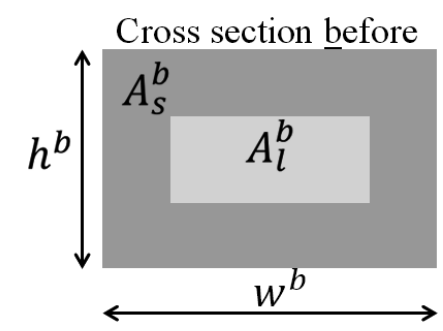

a)

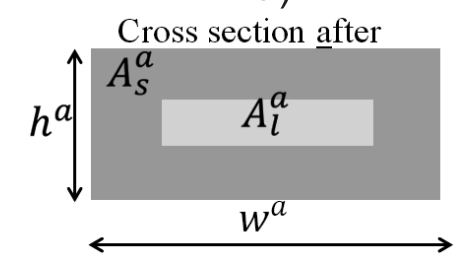

b)

Fig. 2. Schematic view of the strand cross section before and after soft reduction where the areas of solid and liquid are indicated.

In Fig. 2, the corresponding strand cross-sections before and after the height reduction are sketched. The area of the liquid phase, $A_{l}$, and solid phase, $A_{s}$ before and after the soft reduction are marked out. The area of the solid phase in the figure represents both the solid strand shell as well as the solid phase in the mushy zone. In the same way, the area of the liquid phase represents the total liquid area in the center including the liquid in the mushy zone. The height reduction, from $h^{b}$ to $h^{a}$, will create both an elongation and a spread of the cross section from width before, $w^{b}$, to width after, $w^{a}$, as indicated in the figure. It should be noted that the strand cross section area, $A=h \cdot w=A_{l}+A_{s}$, is the sum of the solid and liquid areas

The derivation of the analytical model is based on the following assumptions:

- The model is one-dimensional in the casting direction

- The liquid and solid densities, $\rho_{l}$ and $\rho_{s}$ are constants and independent of temperature and concentration

- No thermal contraction of the strand takes place

- The downward flow velocities of the liquid and solid phase, $v_{l}$, and $v_{s}$, are averages over the strand cross-section

- The cross section of the strand before and after height reduction is planar

The volume flows immediately before and after the deformation in Fig. 1 are $d V^{b} / d t$ and $d V^{a} / d t$, respectively, and can be expressed as: 


$$
\begin{aligned}
& \frac{d V^{b}}{d t}=v_{l}^{b} \cdot A_{l}^{b}+v_{s}^{b} \cdot A_{s}^{b} \\
& \frac{d V^{a}}{d t}=v_{l}^{a} \cdot A_{l}^{a}+v_{s}^{a} \cdot A_{s}^{a}
\end{aligned}
$$

Assuming that no solidification takes place during deformation, the inflow of volume to the deformation zone must be equal to the outflow; thus $d V^{b} / d t=d V^{a} / d t$. This gives the relations between the liquid and solid volumes:

$$
\begin{aligned}
& v_{l}^{b} \cdot A_{l}^{b}=v_{l}^{a} \cdot A_{l}^{a} \\
& v_{s}^{b} \cdot A_{s}^{b}=v_{s}^{a} \cdot A_{s}^{a}
\end{aligned}
$$

Dividing Eq. (3a) by Eq. (3b) gives a useful relationship between the velocities of the liquid and solid phases:

$$
\frac{v_{l}^{b}}{v_{s}^{b}}=\frac{v_{l}^{a}}{v_{s}^{a}} \cdot D
$$

where the deformation constant, $D$, is defined as:

$$
D=\frac{A_{l}^{a} \cdot A_{s}^{b}}{A_{l}^{b} \cdot A_{s}^{a}}
$$

As mentioned in the introduction, it is the velocity difference between liquid and solid phases, expressed as the ratio, $v_{l} / v_{s}$, that causes segregations and pores. The purpose of MSR is to reduce that velocity difference at positions in front of the pinch rollers.

A special case of interest to study is a 'homogeneous deformation' of the strand cross section, here defined by the situation when $D=1$. Then Eq. (4) gives that the velocity ratios before and after deformation will be equal namely, $v_{l}^{b} / v_{s}^{b}=v_{l}^{a} / v_{s}^{a}$. Consequently no change of relative velocities takes place and hence no influence on the segregation and pore formation will occur. This case is illustrated in Figs. 3(a) and 3(b) with the shape of a longitudinal volume element before and after a height reduction. The liquid in the center is deformed in proportion to the solid phase deformation.

It should also be mentioned that the case of $D=1$ in Eq. (5) gives $A_{l}^{a} / A_{l}^{b}=A_{s}^{a} / A_{s}^{b}=A^{a} / A^{b}$. These relationships will later be used in Eq. (6).

The velocity ratio before the deformation zone, $v_{l}^{b} / v_{s}^{b}$, can be reduced if $D<1$ or $A_{l}^{b} / A_{s}^{b}>A_{l}^{a} / A_{s}^{a}$. This is achieved through a larger plastic deformation of the liquid relative to the solid phase, which corresponds to a 'non-uniform deformation'. Figure 3(c) illustrates how a volume element is deformed and that the liquid phase is pushed backward in the direction of the mold. (A flow in the casting direction is not possible due to the closed volume of liquid behind the pinch rollers)

To be able to calculate the influence of MSR on the liquid flow, it is necessary to know how much of the area reduction will contribute to the push-back of liquid. This can be estimated by comparing the deformation of the liquid core with a void/hole closure during plastic deformation. Such data can be found in the literature for rolling and forging cases. However, these cases do not normally take into account the sharp temperature gradient in the material that exists during MSR and how this gradient enhances the void closure ratio.

During MSR, the height reduction is relatively small
Before height reduction

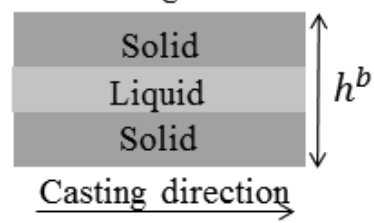

a)

\section{Homogenous deformation}

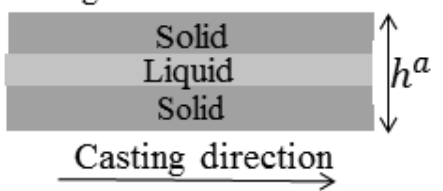

b)

Inhomogeneous deformation Part of liquid pushed backward

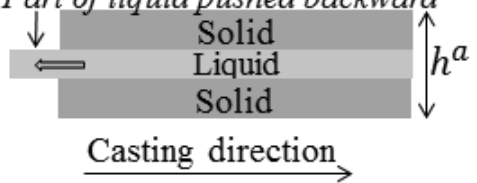

C)

Fig. 3. Shape of strand including liquid and solid phases a) Before height reduction b) After homogenous deformation c) After inhomogeneous deformation.

at each pinch roller. The void closure, here defined as the decrease of the liquid area ratio, $A_{l}^{a} / A_{l}^{b}$, is approximated by a linear expression, Eq. (6) below, where $C$ is a closure constant dependent on different deformation conditions, such as temperature gradients and roller contact area:

$$
\frac{A_{l}^{a}}{A_{l}^{b}}=1-C\left(1-\frac{A^{a}}{A^{b}}\right) .
$$

where $A^{a}$ and $A^{b}$ are the areas of the strand cross section after and before reduction and the term $\left(1-A^{a} / A^{b}\right)$ defines the area reduction. In the case $\mathrm{C}=1$, Eq. (6) is equal to $A_{l}^{a} / A_{l}^{b}=A^{a} / A^{b}$ which corresponds to a 'homogenous deformation' with $D=1$. The void will never close and no push-back of liquid occurs. The push-back of liquid area ratio, $\Delta A_{l}^{\text {Push }} / \mathrm{A}_{1}^{\mathrm{b}}$, that contributes to the decrease of the liquid flow velocity, can now be estimated by using the difference between the cases with $\mathrm{C}>1$ and $\mathrm{C}=1$ in Eq. (6):

$$
\frac{\Delta A_{l}^{\text {Push }}}{\mathrm{A}_{1}^{\mathrm{b}}}=\mathrm{P} \cdot\left(1-\mathrm{A}^{\mathrm{a}} / \mathrm{A}^{\mathrm{b}}\right)
$$

where $\mathrm{P}=\mathrm{C}-1$, is the push-back constant.

In the literature several publications deals with void closure related to area reduction in solid materials. This information can be used to estimate values of C. In the early work of Leduc et al., ${ }^{15)}$ it is reported that rolling slabs with voids gives completely dense material with area reduction ratios between 0.5 to 0.8 . This gives $\mathrm{C}=1.25-2$. Tsuda et $a l .{ }^{16)}$ carried out experiments with plasticine to study how the temperature gradient affects the necessary reduction to close voids. Their results indicate a critical reduction of 0.37 which gives $\mathrm{C}=2.7$. Wallerö ${ }^{17)}$ measured the void closure by Filia-wax experiments giving $\mathrm{C}=1.4-2.6$. Kakimoto et al. ${ }^{18)}$ used a 2D finite element method to calculate a void closure for a rectangular section with a round void. For small area 
reductions, their result gives $\mathrm{C}=2$.

As already mentioned, it is not easy to determine $\mathrm{C}$ in Eq. (6) accurately. Therefore in this analysis two values will be used, namely $\mathrm{C}=2$ and $\mathrm{C}=3$. The corresponding area reduction ratios for complete pore closure are 0.5 and 0.33 , numbers which are in accordance with aforementioned experimental results. In Fig. 4, the liquid area ratio is plotted against area reduction ratios for $\mathrm{C}=1,2$ and 3 and the pushback liquid area ratios for $\mathrm{P}=1$ and 2 . These results will be used later on for calculating the liquid flow velocities.

\subsection{Model of Liquid Flow Velocity due to MSR and Solidification Shrinkage}

The influence of MSR on the liquid flow velocity along the strand can be estimated according to the model proposed by Rogberg. ${ }^{12)}$ A mass balance was set up within the mushy zone considering a volume element $\Delta x \cdot A(x, t)$, outlined in Fig. 5. Here the function $A(x, t)$ represents the cross section of the strand and is allowed to vary with time and distance to reproduce MSR. The net inflow of liquid mass to the volume element is caused by solidification shrinkage and by the deformation of the strand by the pinch rollers. Note that the solidification takes place opposite to the casting direction.

Conservation of mass balance in the volume element gives:

$$
\frac{\partial}{\partial t}\left(A_{s}(x, t) \cdot \rho_{s}+A_{l}(x, t) \cdot \rho_{l}\right)=-\frac{\partial}{\partial x}\left(A_{l}(x, t) \cdot \rho_{l} \cdot v_{l}^{r e l}(x, t)\right)
$$

where $A(x, t)$ is the sum of liquid and solid areas:

$$
A(x, t)=A_{l}(x, t)+A_{s}(\mathrm{x}, \mathrm{t}) \text {. }
$$

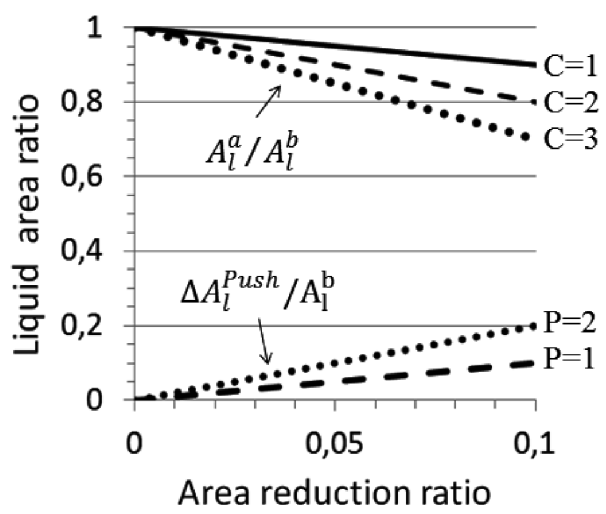

Fig. 4. Liquid area ratio versus the area reduction ratio for different values of C in Eq. (6) and P in Eq. (7).

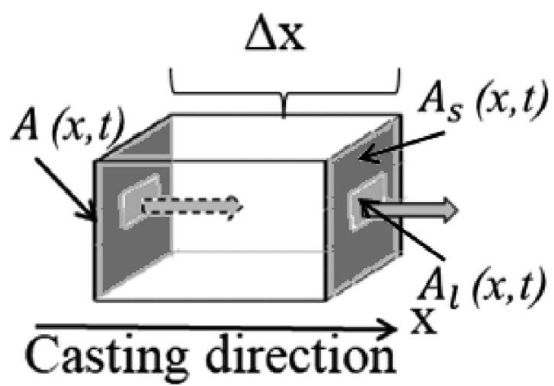

Fig. 5. Definition of variables used in the volume element considered.
The liquid flow velocity is obtained by integrating Eq. (8):

$$
v_{l}^{r e l}(x, t)=-\frac{1}{A_{l}(x, t) \cdot \rho_{l}} \cdot \int_{x_{0}}^{x} \frac{\partial}{\partial t}\left[A_{l}(x, t) \cdot \rho_{l}+A_{s}(x, t) \cdot \rho_{s}\right] d x
$$

The lower integration limit, $x_{o}$, is equal to the point where the center of the strand has just solidified. The upper integration limit corresponds to a distance $x$ from the meniscus. By making the substitution $x^{\prime}=x+v_{\text {cast }} \cdot t$ and also assuming stationary conditions, Eq. (10) transforms into:

$$
\begin{aligned}
& v_{l}^{r e l}\left(x^{\prime}\right)= \\
& -v_{\text {cast }} \cdot \frac{1}{A_{l}\left(x^{\prime}\right) \cdot \rho_{l}} \cdot \int_{M L}^{x^{\prime}} \frac{\partial}{\partial x^{\prime}}\left[A_{l}\left(x^{\prime}\right) \cdot \rho_{l}+A_{s}\left(x^{\prime}\right) \cdot \rho_{s}\right] d x^{\prime}
\end{aligned}
$$

Here, $M L$ is the metallurgical length defined as the distance from the meniscus to the point where the strand center has just solidified. Equation (11) can be integrated directly and after inserting the upper and lower integration limits:

$$
\begin{aligned}
& v_{l}^{r e l}\left(x^{\prime}\right)=-v_{\text {cast }} \cdot \frac{1}{A_{l}\left(x^{\prime}\right) \cdot \rho_{l}} \\
& \cdot\left[A_{l}\left(x^{\prime}\right) \cdot \rho_{l}+A_{s}\left(x^{\prime}\right) \cdot \rho_{s}-A_{l}(M L) \cdot \rho_{l}-A_{s}(M L) \cdot \rho_{s}\right]
\end{aligned}
$$

As $A_{l}(M L)=0$, Eq. (9) gives $A_{s}(M L)=A(M L)$. Using these relations and combining Eqs. (9) and (12) gives the final analytical equation, describing how the liquid flow velocity varies with distance from the meniscus along the strand due to solidification shrinkage and MSR:

$v_{l}^{r e l}\left(x^{\prime}\right)=-v_{\text {cast }} \cdot\left[1-\frac{\rho_{s}}{\rho_{l}}+\frac{\left(A\left(x^{\prime}\right)-A(M L)\right)}{\left(A\left(x^{\prime}\right)-A_{s}\left(x^{\prime}\right)\right)} \cdot \frac{\rho_{s}}{\rho_{l}}\right]$

In the case of no MSR the strand cross section will not vary with the distance from meniscus which gives $A\left(x^{\prime}\right)=$ $A(M L)$. Putting this relation into Eq. (13) gives the following relationship for $x^{\prime}<M L$ :

$$
v_{l}^{r e l}\left(x^{\prime}\right)=v_{\text {cast }} \cdot\left(\frac{\rho_{s}}{\rho_{l}}-1\right)=v_{\text {cast }} \cdot \beta
$$

Equation (14) is the same as Eq. (1), which was expected. During MSR, the term $A_{s}\left(x^{\prime}\right)$ will not be affected in Eq. (13). It is only the part of the liquid area change, $\Delta A_{l}^{\text {Push }}$, that contributes to a push-back of the liquid, that should be used in Eq. (13) to calculate how $A\left(x^{\prime}\right)$ is affected. At each pinch roller $A\left(x^{\prime}\right)$ will decrease with $\Delta A_{l}^{\text {Push }}$. Thus $A\left(x^{\prime}\right)$ will be a stepwise continuous function.

\section{Analysis of the Liquid Flow Velocities by the Analyti- cal Model}

Equation (13) can now be utilized to give a quantitative example of the influence of MSR on $v_{l}^{\text {rel }}\left(x^{\prime}\right)$ for a bloom caster with size $365 \times 265 \mathrm{~mm}$. In this example the pinch rollers are located $17.7 \mathrm{~m}, 19.8 \mathrm{~m}$ and $22 \mathrm{~m}$ from the meniscus. The choices of relevant height reductions, $\Delta h=$ $h^{b}-h^{a}$, and area reductions are selected from actual experiments. The solid area $A_{s}\left(x^{\prime}\right)$ can be estimated by use of the 
Rule of Thumb, which expresses the shell thickness, s, as function of $t^{\prime}=x^{\prime} / v_{\text {cast }}$ :

$$
s=K \sqrt{\frac{x^{\prime}}{v_{\text {cast }}}}
$$

Different interesting cases for analyses are presented in Table 1. Calculation of $A\left(x^{\prime}\right)$ is done by aid of Eq. (7) to first determine normalized values of $\Delta A_{l}^{\text {Push }} / A(M L)$ and then the stepwise changes of $A(p) / A(M L)$, where $p$ marks the position of a pinch roller.

In Fig. 6(a), the influence of different height reductions are plotted at a roller position, $p=19.8 \mathrm{~m}$. The continuous curve shows the case with no MSR. The liquid flow velocity is in this case constant, $0.4 \mathrm{~mm} / \mathrm{s}$ throughout the metallurgical length. The dashed line, corresponding to $\Delta h=5 \mathrm{~mm}$ and $\mathrm{C}=2$, shows an instant decrease of the flow velocity to $0.2 \mathrm{~mm} / \mathrm{s}$. Closer to the meniscus, the velocity gradually increases and at the mold level the velocity is just below 0.4 $\mathrm{mm} / \mathrm{s}$. The reason why the velocity does not approach the value $0.4 \mathrm{~mm} / \mathrm{s}$ is that the strand area after the pinch roller is smaller than the mold area by $\Delta A_{l}^{\text {Push }}$. Consequently the inflow of liquid to the mold will decrease by $\Delta A_{l}^{\text {Push }} \cdot v_{\text {cast }}$. The dotted line shows that at $\Delta h=10 \mathrm{~mm}$ the liquid flow velocity has gone down to almost zero. The flow velocity resumes a constant value, $0.4 \mathrm{~mm} / \mathrm{s}$, from behind the roller position $p=19.8 \mathrm{~m}$ to the crater end position, $M L$.

The effect of an increased inhomogeneous deformation of the liquid area, corresponding to $\mathrm{C}=3$, is shown in Fig. 6(b). The velocity is now even negative for $\Delta h=10 \mathrm{~mm}$ meaning that the flow is directed opposite to the casting direction.

The results in Fig. 6 indicate that both the instant decrease of flow velocity and the duration length, defined as the length corresponding to where velocities are smaller than

Table 1. Input data to Eq. (13) for calculation of the liquid flow velocities, $v_{l}^{r e l}\left(x^{\prime}\right)$, in Figs. 6(a), 6(b) and 7(a), 7(b) for different cases of area reductions, position of pinch rollers and values for the void closure constant, C. $K=25 \mathrm{~mm} /$ $\min ^{0.5}, \rho_{s} / \rho_{l}=1.03, v_{\text {cast }}=0.8 \mathrm{~m} / \mathrm{min}, M L=22.5 \mathrm{~m}$.

\begin{tabular}{|c|c|c|c|c|c|}
\hline $\begin{array}{l}\text { Position } p \\
\mathrm{~m}\end{array}$ & $\begin{array}{c}h^{b}-h^{a} \\
\mathrm{~mm}\end{array}$ & $\begin{array}{c}\text { Area } \\
\text { reduction }\end{array}$ & $A_{l}^{b}(p) / A(M L)$ & $A^{b}(p) / A(M L)$ & Note \\
\hline 19.8 & 0 & 0 & 0.0199 & 1 & $\begin{array}{c}C=2, \\
\text { Fig. } 6(a)\end{array}$ \\
\hline -“- & 5 & 0.0149 & 0.0202 & 1.00030 & -“- \\
\hline -“" & 10 & 0.0286 & 0.0204 & 1.00057 & -“. \\
\hline 19.8 & 0 & 0 & 0.019 & 1 & $\begin{array}{l}\quad \mathrm{C}=3 \text {, } \\
\text { Fig. } 6(\mathrm{~b})\end{array}$ \\
\hline -“- & 5 & 0.0149 & 0.0205 & 1.00059 & -“" \\
\hline -“- & 10 & 0.0286 & 0.0210 & 1.00114 & -“" \\
\hline 17.7 & 5 & 0.0149 & 0.0416 & 1.00061 & $\begin{array}{c}\quad \mathrm{C}=2, \\
\text { Fig. 7(a) }\end{array}$ \\
\hline 19.8 & 5 & -“" & 0.0202 & 1.00030 & -“- \\
\hline 22 & 5 & -“. & 0.0031 & 1.00005 & -“"- \\
\hline 17.7 & 5 & 0.0149 & 0.0416 & 1.00095 & $\begin{array}{c}\quad C=2, \\
\text { Fig. } 7(b)\end{array}$ \\
\hline 19.8 & 5 & -“"- & 0.0202 & 1.00034 & -“" \\
\hline 22 & 5 & -“. & 0.0031 & 1.00005 & -“- \\
\hline
\end{tabular}

$0.33 \mathrm{~mm} / \mathrm{s}(\sim 85 \%$ of $0.4 \mathrm{~mm} / \mathrm{s})$, is approximately proportional to the amount of height reduction.

The individual effect of MSR at positions, $17.7 \mathrm{~m}, 19.8 \mathrm{~m}$ and $22 \mathrm{~m}$, can be seen in Fig. 7(a) for the case of $\Delta h=5 \mathrm{~mm}$ and $\mathrm{C}=2$. The results have been plotted in the same diagram to enable a comparison. The maximum velocity decrease is about the same at each position, but the effect of MSR on the results differs. The upturn in flow velocity is slower for pinch roller positions closer to the meniscus. The reason is that the liquid area is larger and hence the $\Delta A_{l}^{\text {Push }}$ value is larger. The duration length is $5.7 \mathrm{~m}, 1.9 \mathrm{~m}$ and $1.1 \mathrm{~m}$ for the pinch roller positions $17.7 \mathrm{~m}, 19.8 \mathrm{~m}$ and $22 \mathrm{~m}$ respectively. If the target of MSR is to obtain the same duration length at each pinch roller, then the height reduction has to increase successively closer to the crater end, $M L$.

The effect of applying MSR at the same time for all 3 positions with $\Delta h=5 \mathrm{~mm}$ and $\mathrm{C}=2$ can be seen in Fig. 7(b). The velocity at the last pinch roller at $p=22 \mathrm{~m}$ has gone down to the same value, $0.2 \mathrm{~mm} / \mathrm{s}$, as in Fig. 7(a). The influence on the velocity from the height reduction at the last pinch roller will be superimposed on the instant decrease of velocity at the first and second pinch rollers. The effect is clearly visible at $p=17.7 \mathrm{~m}$ where the velocity has gone down to $0.1 \mathrm{~mm} / \mathrm{s}$, somewhat lower than the value of 0.2 $\mathrm{mm} / \mathrm{s}$ in Fig. 7(a). To have a more even reduction of the flow velocities along the reduction zone, the height reduction should increase closer to the crater end.

The dotted line in Fig. 7(b) shows how the term $A\left(x^{\prime}\right) /$

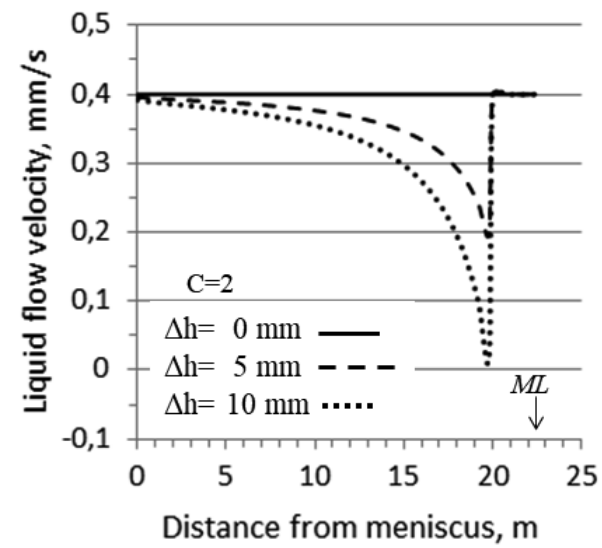

a)

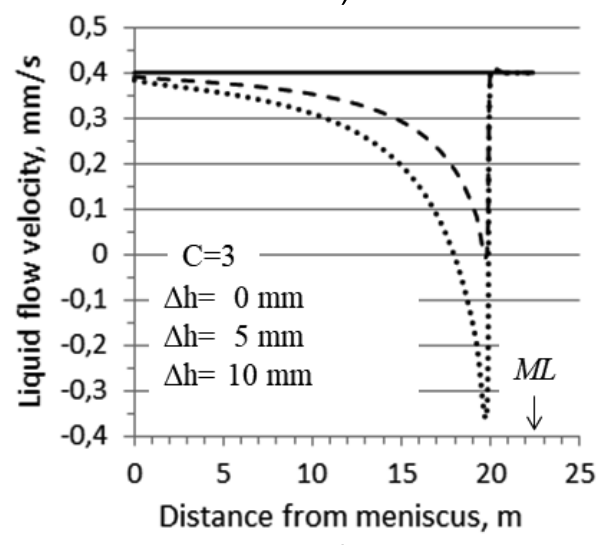

b)

Fig. 6. a) Liquid flow velocities, $v_{l}^{\text {rel }}\left(x^{\prime}\right)$ for the case of $\mathrm{C}=2$, MSR at position $p=19.8 \mathrm{~m}$ from meniscus and different height reductions, $\Delta h$. b) The same parameters but $\mathrm{C}=3$. 
$A(M L)$, i.e. the relative change of strand area corresponding to the push-back of liquid, changes due to the height reduction.

By putting $v_{l}^{\text {rel }}\left(x^{\prime}\right)=0$ in Eq. (13) gives how $A\left(x^{\prime}\right) / A(M L)$ should vary from $x^{\prime}$ to $M L$, a kind of an ideal reduction along the strand length to receive no relative velocity differences between solid and liquid. As pointed out earlier, the decrease of strand area will only decrease the liquid area:

$$
\frac{A\left(x^{\prime}\right)}{A(M L)}=\frac{\rho_{s}}{\rho_{l}}-\frac{A_{s}\left(x^{\prime}\right)}{A(M L)} \cdot\left[\frac{\rho_{s}}{\rho_{l}}-1\right]
$$

It is interesting to note that $A\left(x^{\prime}\right)$ is indirectly dependent

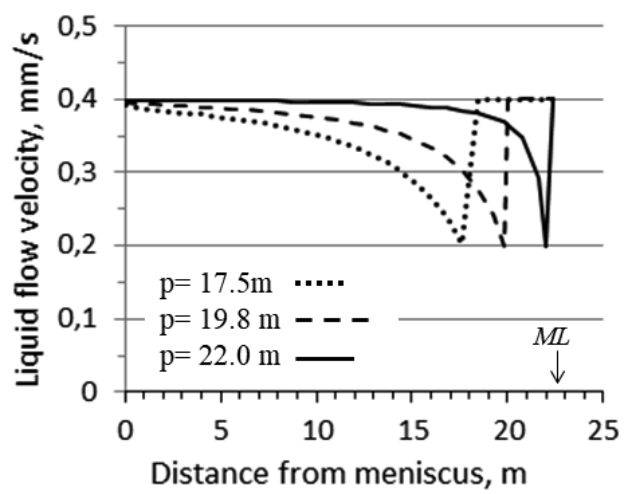

a)

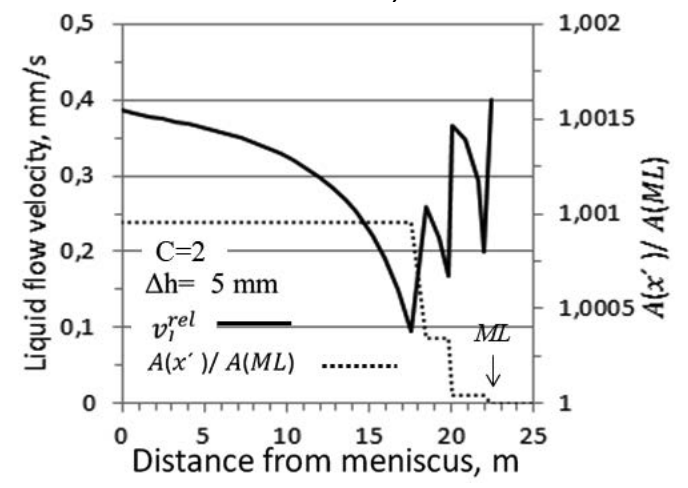

b)

Fig. 7. Effect of MSR on the liquid flow velocities, $v_{l}^{\text {rel }}\left(x^{\prime}\right)$. a) Individual effect of MSR at each pinch roller b) MSR applied at the same time. Dotted line shows ratio of $A\left(x^{\prime}\right) /$ $A(M L)$.

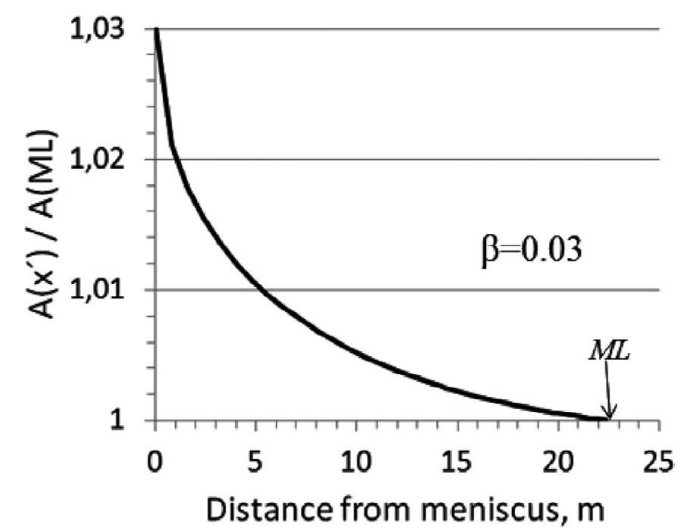

Fig. 8. Ideal area reduction along the strand from the meniscus to the crater end, $M L$, giving zero fluid flow due to solidification shrinkage. on the casting speed by $M L$. Equation (16) is plotted in Fig. 8. For example, the curve in Fig. 8 shows that to obtain zero liquid flow velocities between the distances $15 \mathrm{~m}$ to $M L$, the value of $A\left(x^{\prime}\right) / A(M L)$ must decrease from the value 1.002 to 1 .

Taking the derivate of Eq. (16) gives the reduction gradient that exactly compensates for the solidification shrinkage:

$$
\frac{d A\left(x^{\prime}\right)}{d x^{\prime}}=-\left[\frac{\rho_{s}}{\rho_{l}}-1\right] \cdot \frac{d A_{s}}{d x^{\prime}}
$$

\section{MSR Experiments}

Experiments with MSR were carried out on a bloom caster, size $365 \times 265 \mathrm{~mm}$. The main machine data is given in Table 2 and, in particular, it includes distances from the meniscus to the pinch rollers. The layout of the straightening and withdrawal unit is displayed in Fig. 9. A gauge device was installed at each pinch roller to measure the height carefully.

Tests were performed on a high carbon steel grade with the objective of reducing the centre macrosegregation and on a stainless steel grade, type AISI316, with the objective of reducing centre porosity. Compositions of the alloys are listed in Table 3. The casting speed was $0.8 \mathrm{~m} / \mathrm{min}$ for both steel grades.

For the carbon steel, a number of MSR trials with different combinations of height reduction were performed.

Table 2. Basic data for the bloom caster.

\begin{tabular}{ll}
\hline - 3 strand curved machine. & - Withdrawal unit: 3 pinch \\
- Bloom size $365 \times 265 \mathrm{~mm}, 265 \times 265 \mathrm{~mm}$ & $\begin{array}{l}\text { and unbending rollers } \\
\text { located at R } 1=17.7 \mathrm{~m},\end{array}$ \\
- Radius: $12 \mathrm{~m}$ & $\begin{array}{l}\mathrm{R} 2=19.8 \mathrm{~m} \text {, and } \mathrm{R} 3=22 \mathrm{~m} \\
\text { from meniscus }\end{array}$ \\
- Casting speed 0.6 to $1.2 \mathrm{~m} / \mathrm{min}$ &
\end{tabular}

Table 3. Composition of tested steel grades in weight $\%$.

\begin{tabular}{cccccc}
\hline Steel grade & $\mathrm{C}$ & $\mathrm{S}$ & $\mathrm{Cr}$ & $\mathrm{Ni}$ & $\mathrm{Mo}$ \\
\hline High Carbon & 0.76 & 0.01 & - & - & - \\
Stainless & $<0.03$ & 0.025 & 17 & 11.5 & 2.1 \\
\hline
\end{tabular}

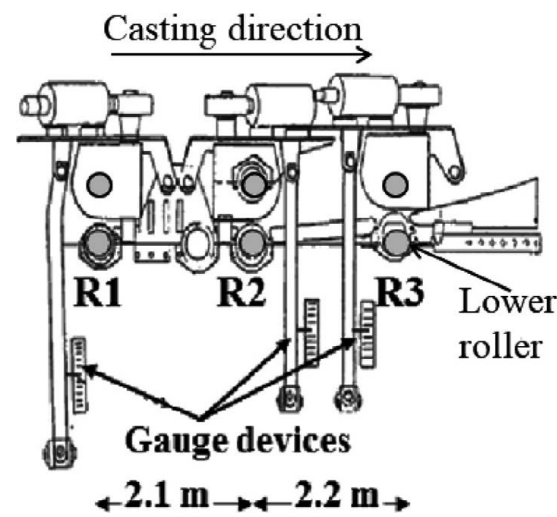

Fig. 9. Straightening and withdrawal unit with pinch rollers R1, R2 and R3. Gauge devices for measurements of strand height reductions are indicated. 
Bloom samples, length $\sim 300 \mathrm{~mm}$, were taken out to evaluate the spread and elongation. The samples were cut perpendicular and parallel to the strand centre axis, ground for visual observation of the centre porosity and then used for sulphur prints. The centre segregation ratio was analysed on chips from drilling $\sim 15$ holes along the bloom centre axis with drill diameter $\varnothing=5 \mathrm{~mm}$. Thus, it was possible to select the maximum segregation ratio, $C_{\max } / C_{0}$, and to calculate the average centre segregation, $C_{a v} / C_{0}$.

MSR was applied on stainless steel primarily to reduce the centre porosity with the objective of improving the centre soundness in rolled bars. Blooms cast without and with MSR of $6 \mathrm{~mm}$ (2.3\% height reduction) were further rolled to bars with diameter $\varnothing=193 \mathrm{~mm}$, corresponding to an elongation of 3.3 or an area reduction of 0.70 . After peeling, the bars were ultra-sonic tested, US-tested. The smallest pore area, which could be detected by this procedure, was 0.2 $\mathrm{mm}^{2}$. During testing the bars were rotated to detect all pores from different probe angles. It should be mentioned that this method records only the total pore area. Consequently, it was not possible to distinguish between single large pores and clusters of pores.

\section{MSR Results}

\subsection{Carbon Steel Grade}

Table 4 summarizes trials and results for the high carbon steel grade. Here, it should be mentioned that a fully columnar structure was obtained for all trials.

The sulphur print in Fig. 10(a) is the reference trial 1 without MSR. The centre is heavily segregated with single

Table 4. Soft reduction trials on a high carbon steel grade. Bloom size $365 \times 265 \mathrm{~mm}$

\begin{tabular}{|c|c|c|c|c|c|c|c|}
\hline \multirow[b]{2}{*}{ Trial } & \multicolumn{4}{|c|}{ Height reduction, mm } & \multicolumn{2}{|c|}{ Segregation ratio } & \multirow{2}{*}{$\begin{array}{l}\text { Visual } \\
\text { porosity }\end{array}$} \\
\hline & $\mathrm{R} 1$ & $\mathrm{R} 2$ & $\mathrm{R} 3$ & Tot & $C_{a v} / C_{0}$ & $C_{\max } / C_{0}$ & \\
\hline 1, ref & 0 & 0 & 0 & 0 & 1.30 & 1.60 & Yes \\
\hline 2 & 7 & 3.5 & 5.5 & 16 & 1.11 & 1.18 & No \\
\hline 3 & 7 & 0 & 0 & 7 & - & - & Yes \\
\hline 4 & 7 & 3.5 & 0 & 10.5 & 1.17 & 1.39 & No \\
\hline 5 & 3.5 & 1.5 & 5.5 & 10.5 & 1.16 & 1.30 & Small \\
\hline
\end{tabular}

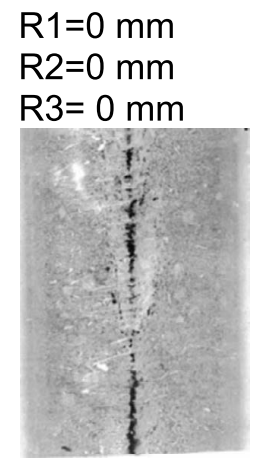

a)

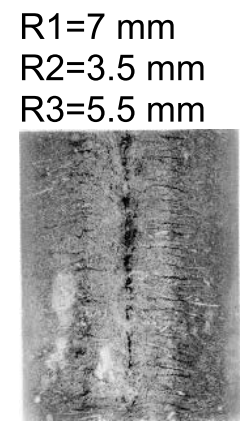

b)

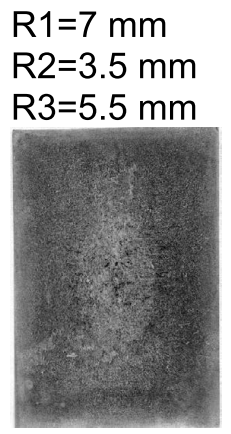

c)
Fig. 10. Sulphur prints from longitudinal and cross section with corresponding height reductions. a) Reference Trial 1 b) Trial 2. c) Trial 2 cross section.
V-segregates appearing at certain intervals. The porosity is clearly visible and the maximum segregation ratio is as high as 1.60 .

In trial 2 , the total height reduction was $16 \mathrm{~mm}$. The corresponding sulphur print can be seen in Fig. 10(b). Severe half-way cracks occur at distances between 28-60 mm from the centre. The orientation of the crack plane is perpendicular to the centre axis and the cracks are created by the elongation of the strand. The sulphur print from the cross section, Fig. 10(c), shows just small signs of cracks, probably with the same origin as in Fig. 10(b). Consequently, no cracks could be detected in a plane oriented parallel to the central axis. The maximum centre segregation ratio decreased to the value 1.18 from the reference value 1.60 .

In trial 3, MSR was only performed at R1. The sulphur print in Fig. 11(a) shows that cracks appear at the same position as in Fig. 10(b). The conclusion is that a $7 \mathrm{~mm}$ height reduction at $\mathrm{R} 1$ gives too long an elongation and that it will give rise to transversal cracks.

In trial 4, the amount of height reduction at R1 and R2 was the same as in trial 2. However, no reduction was carried out at R3. In comparison with trial 2, the maximum segregation ratio increased by 0.21 units to a value of 1.39 , which in its turn is 0.21 units below the reference value 1.60. The sulphur print in Fig. 11(b) shows cracks but no visual porosities could be seen on the ground sample. The conclusion is that MSR at R3, closer to the crater end, has about the same effect to reduce segregations as MSR at only $\mathrm{R} 1+\mathrm{R} 2$.

In the last trial 5, the height reduction was reduced to 3.5 $\mathrm{mm}$ at R1 with the intention to avoid cracks. The sulphur print in Fig. 11(c) shows almost no half-way cracks with just small visual centre porosities. The maximum segregation ratio obtained was 1.30 , substantially lower than the reference value of 1.60 with no MSR.

Results from measurements of height and width on bloom samples are presented in Fig. 12(a). No bulging of the parallel sides could be seen. In Fig. 12(b), the elongation = $\ln \left(\frac{h^{b} \cdot w^{b}}{h^{a} \cdot w^{a}}\right)$ and the width spread $=\ln \left(\frac{w^{a}}{w^{b}}\right)$ are plotted. The ratio between the elongation and spread is $\sim 2.5$. The results can be used to calculate the area reduction which is needed as input to Eq. (13).

The data in Fig. 12(b) can be utilized to explain the devel-

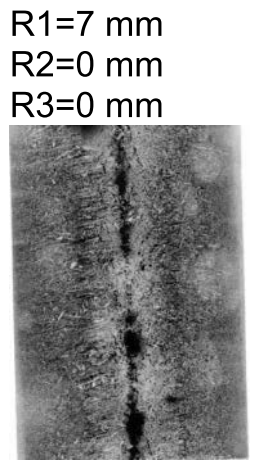

a)

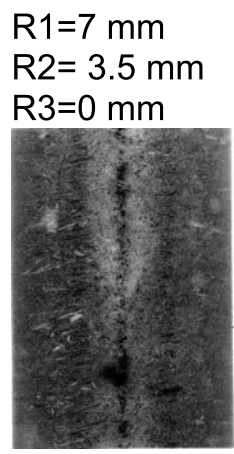

b)

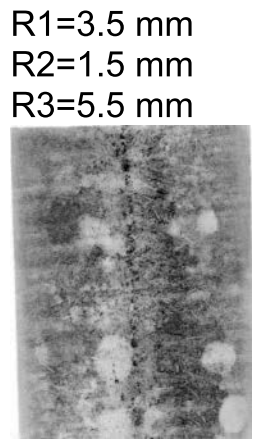

c)
Fig. 11. Longitudinal sulphur prints with corresponding height reduction at each pinch roller on top a) Trial 3. b) Trial 4. c) Trial 5 . 
opment of cracks due to MSR. A height reduction of $7 \mathrm{~mm}$ at R1 corresponds to an elongation of $\sim 2 \%$ and a spread of $0.8 \%$. This elongation caused the cracks shown in Fig. 10(b), but no longitudinal cracks due to the spread in Fig. 10(c). By reducing the height reduction at $\mathrm{R} 1$ to $3.5 \mathrm{~mm}$, corresponding to $0.9 \%$ elongation, almost no cracks were visible. It seems that at $\mathrm{R} 1$ the reduction should be below $1 \%$ to avoid cracks. A reduction of $5 \mathrm{~mm}$ at $\mathrm{R} 3$, corresponding to $1.3 \%$ elongation gave no cracks. At R3 the liquid fraction is smaller in the centre compared to the position at $\mathrm{R} 1$ and that seems to have had a positive effect on the crack propensity. The conclusion is that the steel grade can sustain a higher elongation without cracking when the liquid fraction is smaller in the mushy zone. Regarding the influence of spread no single experimental value exceeded $1 \%$ and that could explain the absence of longitudinal cracks.

The mathematical model shows that the liquid flow velocity increases to the value corresponding to no MSR behind the last pinch roller. A relevant question is how long the mushy zone has to be in the centre behind the last pinch roller to create the necessary downward liquid flow velocities that in its turn start to create centre segregation? To check this, 6 longitudinal samples were taken from the front end of a bloom with a size of $265 \times 265 \mathrm{~mm}$. Figure 13 shows sulphur prints from a high carbon steel grade cast at a casting speed of $0.6 \mathrm{~m} / \mathrm{min}$. The dummy bar head, as well as pieces of the cooling scrap, are visible at the bottom of sulphur print 1 . Regular bridging and pronounced centre segregation starts to occur in sulphur print 6 at a distance of about $1.2 \mathrm{~m}$ cast length or $\sim 4$ times the height of the bloom. The conclusion is that the last pair of pinch rollers should

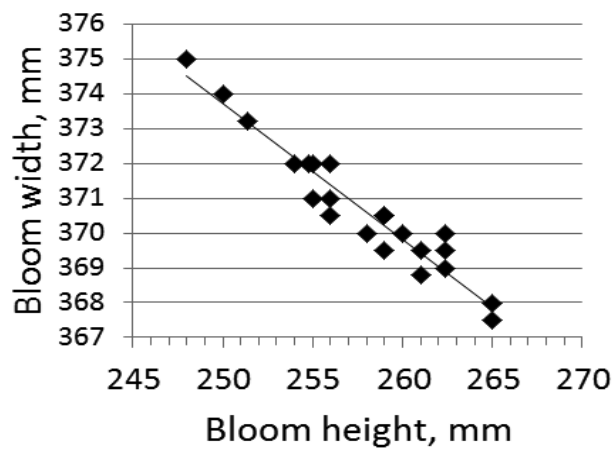

a)

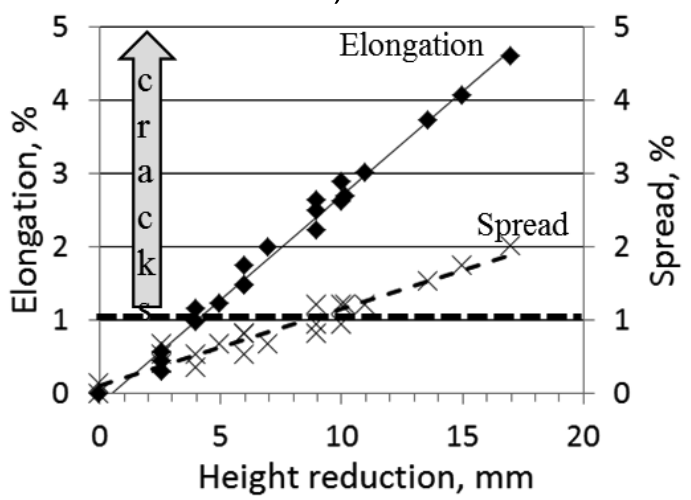

b)

Fig. 12. Measurement results of bloom height and width after MSR. b) Calculated elongation and spread vs height reduction. be located at a distance no greater than 4 times the bloom height from the crater end to reduce the risk of deterioration of bridging and centre segregation.

\subsection{Stainless Steel}

Figure 14(a) shows a longitudinal ground bloom sample from a reference heat cast without MSR. The centre porosity is more or less continuous throughout the central axis. Another reference cast can be seen in Fig. 14(b). The sample has been etched and the secondary structure is visible. Even in this case, the centre porosity is more or less continuous.

Figure 14(c) shows an etched bloom sample from MSR with a total height reduction of $5 \mathrm{~mm}$ for all the three pinch rollers. No visual porosity could be discerned, nor any halfway cracks.

US-results from a reference bar with diameter $\varnothing=193$ $\mathrm{mm}$ originating from a bloom cast without MSR are shown as a dashed line in Fig. 15. A continuous porosity exists throughout the length of the bar. The area of pores corresponds to about $6.2 \mathrm{~mm}^{2}$. The diamonds show US-results on bars, $\varnothing=193$, from a bloom cast with a total height reduction of $6 \mathrm{~mm}$. The porosity appears at only certain intervals

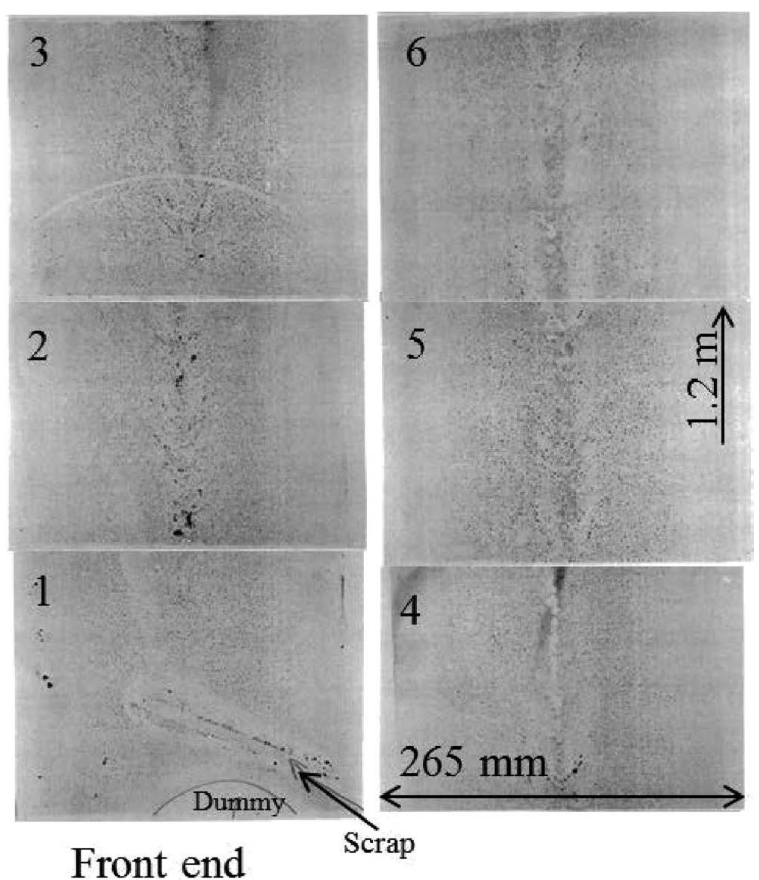

Fig. 13. Sulphur prints from front end of bloom at casting speed of $0.6 \mathrm{~m} / \mathrm{min}$. Regular bridging starts after $\sim 1.2 \mathrm{~m}$ cast length.

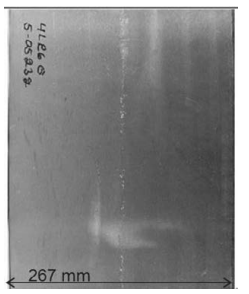

a)

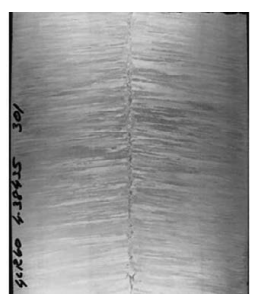

b)

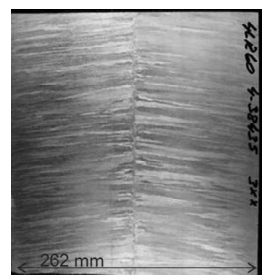

c)
Fig. 14. Longitudinal samples AISI316. a) Reference samples with ground surface showing centre porosity. b) Reference sample with etched surface. c) Etched sample, $\Delta \mathrm{h}=5 \mathrm{~mm}$, with no visual porosity or cracks. 


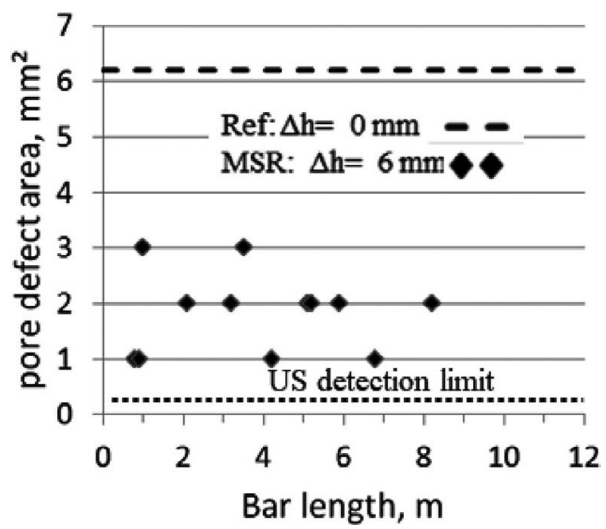

Fig. 15. US testing results on stainless steel bars, $\varnothing=193 \mathrm{~mm}$, from blooms with and without MSR.

along the bar and the pore areas vary between $1-3 \mathrm{~mm}^{2}$. The number of defects per meter of bar length is 0.8 , which corresponds to a bloom length of $0.24 \mathrm{~m}$. Obviously, most of the more or less continuous centre porosity in the cast bloom has been reduced to sizes below the US-detection limit. The results indicate that only a small height reduction causes a pronounced positive effect on the centre soundness of the rolled bars. It should here also be mentioned that the relatively small height reduction obtained was due to limiting available pinch forces, as the yield strength for stainless steels is higher than for carbon steels.

\section{Discussion}

One interesting question is how the process parameter, reduction gradient, should be specified to fully compensate for the solidification shrinkage. Miyazawa and Ohashi ${ }^{19)}$ presented a theoretical calculation of how the minimum necessary gradient of squeezing, $\Delta h / \Delta x^{\prime}$, should vary from the meniscus to $M L$ with the condition 'without forward slip of solidified shell'. At a distance of $2.6 \mathrm{~m}$ from $M L$, corresponding to pinch roller R2 in this paper, they calculated a value of $\Delta h / \Delta x^{\prime} \sim 0.18 \mathrm{~mm} / \mathrm{m}$ for a strand width of $300 \mathrm{~mm}$ (no strand thickness or densities are given in the paper). Their result also says that $\Delta h / \Delta x^{\prime}=0$ at $M L$ in contradiction to results in Fig. 8, where the slope of the curve at $M L$ is not zero.

These theoretical results can be compared to the calculated results for the size $365 \times 265 \mathrm{~mm}$ in this paper. The necessary gradient can be estimated from Eq. (17) and Fig. 12 (a) to be $\Delta h / \Delta x^{\prime}=d A\left(x^{\prime}\right) / d x^{\prime} \cdot \Delta h / \Delta A=26 \mathrm{~mm}^{2} / \mathrm{m} \cdot 0.0027$ $\mathrm{mm} / \mathrm{mm}^{2}=0.07 \mathrm{~mm} / \mathrm{m}$. This is less than half of the value indicated by Miyazawa and Ohashi, but of the same order of magnitude.

The reduction gradient, $\Delta h / \Delta x^{\prime}$, in trial 5 , has the value of $10.5 \mathrm{~mm} / 4.3 \mathrm{~m}=2.4 \mathrm{~mm} / \mathrm{m}$ and is 13 to 34 times higher than the theoretical calculated values above. The main explanation for the big differences between theoretical values and practical results can be attributed to that only a part of the height reduction during the trials contributes to a push-back of liquid.

Ogibayashi et al. ${ }^{5)}$ performed MSR trials on a bloom with size $300 \mathrm{~mm} \times 400 \mathrm{~mm}$. The pinch rollers were of convex-shape. They found that the optimum rate for the process parameter, reduction rate, should be in the range of

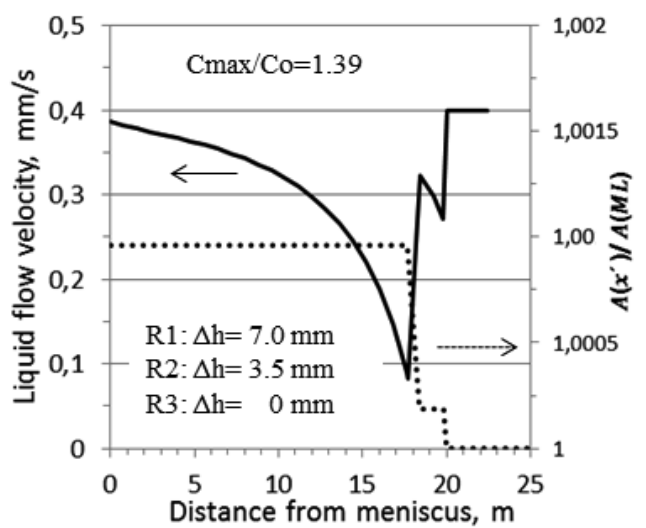

a)

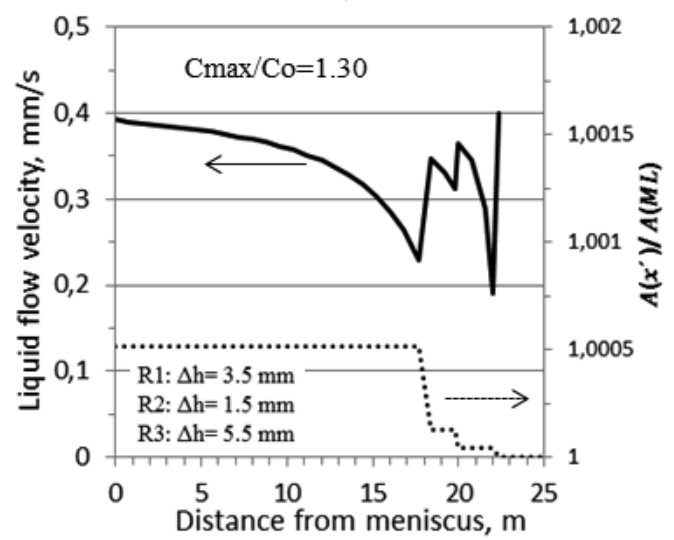

b)

Fig. 16. Calculated liquid flow velocity versus distance from meniscus. a) Trial 4 b) Trial 5.

1.05 to $1.5 \mathrm{~mm} / \mathrm{min}$. These values can be compared to the most promising result in trial 5 , in this report, which gives a value of $1.95 \mathrm{~mm} / \mathrm{min}$. This value is outside the optimum range and indicates the difficulties when comparing results between different continuous casting machines by use of the process parameter reduction rate.

It is interesting to compare trial 4 with trial 5 given in Table 4. The total height reduction was $10.5 \mathrm{~mm}$ in both cases. The main differences between the trials are the height reduction at $\mathrm{R} 3$ and the different amounts of reduction at $\mathrm{R} 1$. The corresponding liquid flow velocities were calculated and the results are presented in Fig. 16. The total amount of the push-back of liquid, $\Delta A_{l}^{\text {Push }} / A(M L)$, is 1.001 in a) but lower in b), 1.0005. Even so the maximum segregation ratio is higher in a) compared to b). Comparing the segregation ratios the effect of reduction at $\mathrm{R} 3$ cannot be compensated for by increasing the reduction at $\mathrm{R} 1$. It seems that the reduction at $\mathrm{R} 3$ is just as important as the reduction at $\mathrm{R} 1$. The results also indicate that the center segregation develops over quite a long distance along the mushy zone center of the bloom.

The results show that, by applying MSR at only one pinch roller, a substantial reduction of the centre segregation and porosity can be obtained. It would be beneficial to do the reduction at a position close to the crater end where the cracking propensity is lower. This is a possibility that can be utilized in CC-machines that are not equipped with a modern reduction zone with several pinch rollers. In this situation, the casting speed must be kept as constant as possible in order to achieve a stable metallurgical length. 
Internal crack formation caused by MSR is an inherent serious problem and a restriction. For the carbon steel grade, transverse cracks developed at R1 with a value of $\Delta \mathrm{h}=7 \mathrm{~mm}$ corresponding to an elongation of $1.9 \%$ in Fig. 12(b). Decreasing the height reduction to a value of $\Delta \mathrm{h}=3.5$ $\mathrm{mm}$, corresponding to a $0.86 \%$ elongation, gave almost no cracking. At R3 no cracks developed at $\Delta \mathrm{h}=5 \mathrm{~mm}$, corresponding to a $1.3 \%$ elongation. This indicates that the crack propensity decreases closer to the crater end position, in accordance to results reported by Sediako et al. ${ }^{9)}$ Also, Won et al. ${ }^{20)}$ performed experiments in a hot tensile testing machine and found that the critical strain is $1 \%$ for a steel grade with 0.65 weight $\%$ carbon content, which is in agreement with the results in this study.

The results in Fig. 7(a) show that the effect of MSR, expressed as the duration length, decreases with a higher fraction value of solid, $f_{s}$, in the bloom centre. From these results, it can be concluded that the distances between the pinch rollers should decrease closer to crater end. At the same time, the amount of reduction should increase. Also, pinch rollers with large diameters should have a positive effect for a given height reduction in the way that the spread will be larger and the elongation smaller, conditions which favour the avoidance of inner crack formation.

\section{Conclusions}

The main conclusions can be summarised as follows:

- Using the developed analytical mathematical model, it is possible to estimate quantitatively the influence of MSR and solidification shrinkage on the average downward liquid flow velocity in the mushy zone

- Calculated results show that the amount of height reduction and distances between the pinch rollers should decrease closer to the crater end to obtain a more even reduction of the flow velocity along the reduction zone.

- Practical results indicate that the centre segregation in continuously cast high carbon steels develops and gradually increases in the mushy zone over a longer distance

- The last pair of pinch rollers should be located at a distance no greater than 4 times the bloom height from the crater end to reduce the risk of deterioration of bridging and centre segregation.

- For high carbon steel grades, the elongation of the bloom due to MSR should be kept below $1 \%$ to avoid internal cracking

- For stainless steels, type AISI 316, just a small height reduction of $2.2 \%$ gives a substantial decrease of centre porosity in cast blooms

\section{Nomenclature}

$C_{0}$ : initial alloy composition, $\mathrm{w} \%$

$\mathrm{C}_{a v}$ : average concentration, $\mathrm{w} \%$
$C_{\max }: \quad$ maximum concentration, $\mathrm{w} \%$

$A$ : area cross section of strand

$A^{b}, A^{a}$ : cross section of strand before and after MSR

$A_{l}^{b}, A_{l}^{a}$ : liquid area before and after MSR

$A_{s}^{b}, A_{s}^{a}$ : $\quad$ solid area before and after MSR

$\Delta A_{l}^{\text {Push }}$ : push-back of liquid area

$h$ : height of strand

$h^{b}, h^{a}$ : height before and after MSR

$w$ : width of strand

$w^{b}, w^{a}$ : width before and after MSR

$V^{b}, V^{a}$ : volume before and after MSR

$v_{\text {cast }}$ : casting speed

$v_{l}^{b}, v_{l}^{a}$ : liquid flow velocity before and after MSR

$v_{s}^{b}, v_{s}^{a}$ : Solid flow velocity before and after MSR

$v_{l}^{r e l}$ : Liquid flow velocity relative to the solid phase

$x, x^{\prime}:$ distance

$p$ : Distance to pinch roller position, $\mathrm{m}$

$t$ : time

$\rho_{l}, \rho_{s}: \quad$ liquid and solid density

$\beta:$ solidification shrinkage, $\left(\frac{\rho_{s}}{\rho_{l}}-1\right)$

$M L$ : metallurgical length, $\mathrm{m}$

$D$ : deformation constant

$C$ : closure constant

$P$ : push-back constant

$K$ : solidification rate constant

\section{REFERENCES}

1) Y. Chen, G. Li, S. Yang and M. Zhu: J. Iron Steel Res. Int., 14 (2007), 13.

2) C. Moon, K. Oh, J. Lee and S. L. Y. Lee: ISIJ Int., 52 (2012), 1266.

3) C. Ji, S. Luo and M. Zhu: ISIJ Int., 54 (2014), 504.

4) M. Wu, J. Domitner and A. Ludwig: Metall. Mater. Trans. A, 43 (2012), 945 .

5) S. Ogibayashi, M. Uchimura, K. Isobe, H. Maede, Y. Nishihara and S. Sato: Proc. 6th Int. Iron and Steel Cong. (IISC), ISIJ, Tokyo, (1990), 271.

6) K. Ayata, S. Koyama, H. Nakata, S. Kawasaki, K. Ebina and T. Hata: Proc. 6th Int. Iron and Steel Cong. (IISC), ISIJ, Tokyo, (1990), 279.

7) Y. Chen, C. Ji, M. Zhu and G. Wu: J. Iron Steel Res., 17 (2010), 31.

8) R. Thome and K. Harste: ISIJ Int., 46 (2006), 1839.

9) D. Sediako, O. Sediako and K. Lin: Can. Metall. Q., 38 (1999), 377.

10) K. Liu and J. Zhang: Adv. Mater. Res., 482-484 (2012), 859.

11) K. Miyazawa and K. Schwerdtfeger: Arch. Eisenhüttenwes., 52 (1081), 415.

12) B. Rogberg: Ph.D. thesis, KTH Royal Institute of Technology, Sweden, (1982).

13) T. Kajitani, J. Drezet and M. Rappaz: Metall. Mater. Trans. A, 32 (2001), 1479.

14) G. Engström, H. Fredriksson and B. Rogberg: Scand. J. Metall., 12 (1983), 3.

15) L. Leduc, T. Nadarajah and C. M. Sellars: Met. Technol., 7 (1980), 269.

16) O. Tsuda, A. Mizuta, Y. Yamaguchi, H. Osuna and H. Yagaki: Tetsuto-Hagané, 67 (1981), S369.

17) A. Wallerö: J. Mech. Work. Technol., 12 (1985), 233.

18) H. Kakimoto, T. Arikawa, Y. Takahashi, T. Tanaka and Y. Imaida: J. Mater. Process. Technol., 210 (2010), 415.

19) K. Miyazawa and T. Ohashi: Trans. Iron Steel Inst. Jpn., 26 (1986), 243.

20) Y. Won, T. Yeo, D. Seol and K. Oh: Metall. Mater. Trans. B, 31 (2000), 779. 